\title{
Propeller and inflow vortex interaction: vortex response and impact on the propeller performance
}

\author{
Yannian Yang $^{1}{ }^{1} \cdot$ Teng Zhou $^{1} \cdot$ Andrea Sciacchitano $^{1} \cdot$ Leo Veldhuis $^{1} \cdot$ \\ Georg Eitelberg ${ }^{1}$
}

Received: 4 February 2016/Revised: 25 May 2016/Accepted: 10 June 2016/Published online: 23 June 2016

(c) The Author(s) 2016. This article is published with open access at Springerlink.com

\begin{abstract}
The aerodynamic operating conditions of a propeller can include complex situations where vorticity from sources upstream can enter the propeller plane. In general, when the vorticity enters in a concentrated form of a vortex, the interaction between the vortex and blade is referred to as blade-vortex interaction or BVI. The interaction may affect the propeller performance as well as its noise production. In the present paper, investigations of the interaction of a wing tip vortex generated by a lifting surface upstream of the rotor plane and an eight-bladed propeller are reported. Utilizing two ends of an upstream wing with non-symmetrical airfoil, the rotation of the incoming vortex could be made to co-rotate or to contra-rotate with the propeller. The ensuing velocity fields were quantified with the help of particle image velocimetry (PIV), and the propeller performance was evaluated with the help of a rotating shaft balance (RSB) mounted on the propeller shaft. The results describe the displacement of the vortex core, as it moves through the rotor plane as well as the positive effect on the thrust and torque of the contra-rotating vortex and the opposite of it in the case of the co-rotating vortex. The current research could be applied to analyse the influence of the incoming vortex on the propeller, e.g., ground vortex, tip vortex shed from a control surface, etc.
\end{abstract}

Keywords Vortex flow B Blade vortex interaction

This paper is based on a presentation at the CEAS Air and Space Conference 2015, September 7-11, Delft, The Netherlands.

Yannian Yang

Y.Yang-1@tudelft.nl

1 Delft University of Technology, Kluyverweg 1, 2629 HS Delft, The Netherlands

\author{
List of symbols \\ $D \quad$ Diameter of propeller, $\mathrm{m}$ \\ $J \quad$ Advance ratio of propeller \\ $Q \quad$ Torque of propeller, $\mathrm{Nm}$ \\ $Q_{C} \quad$ Torque coefficient \\ $R \quad$ Radius of propeller, $\mathrm{m}$ \\ $T \quad$ Thrust of propeller, $\mathrm{N}$ \\ $T_{C} \quad$ Thrust coefficient \\ $U_{\mathrm{eq}} \quad$ Equilibrium inflow velocity of propeller, $\mathrm{m} / \mathrm{s}$ \\ $U_{\text {rad }} \quad$ Radial velocity, $\mathrm{m} / \mathrm{s}$ \\ $U_{\tan } \quad$ Tangential velocity, $\mathrm{m} / \mathrm{s}$ \\ $U_{\infty} \quad$ Free stream velocity, $\mathrm{m} / \mathrm{s}$ \\ $n \quad$ Propeller rotating speed, round/s \\ $r^{-} \quad$ Vortex radius in front of the propeller disk, \\ normalized by propeller radius \\ $r^{+} \quad$ Vortex radius behind the propeller disk, \\ normalized by propeller radius \\ $\Gamma_{\text {imp,cor }}$ Circulation in the core of the wing tip vortex, \\ normalized by $U_{\mathrm{eq}} * D$ \\ $\omega \quad$ Vorticity, $\mathrm{rad} / \mathrm{s}$
}

\section{Introduction}

The blade vortex interaction (BVI) phenomenon is well known in the community of turbomachinery and rotorcraft aerodynamics. For an aircraft propeller, one or more vortices can arise from a ground plane and impinge on the blades during ground operation [1], as shown on the lefthand side of Fig. 1. For the counter-rotating open rotors (CROR), the tip vortex shed form the front rotor may impinge onto the aft rotor, as the two rotors have the same radius [2] or as the rotors operate at an angle of attack [3]. For a turbofan engine, besides the ground vortex impingement [4], the blade vortex interaction can also 

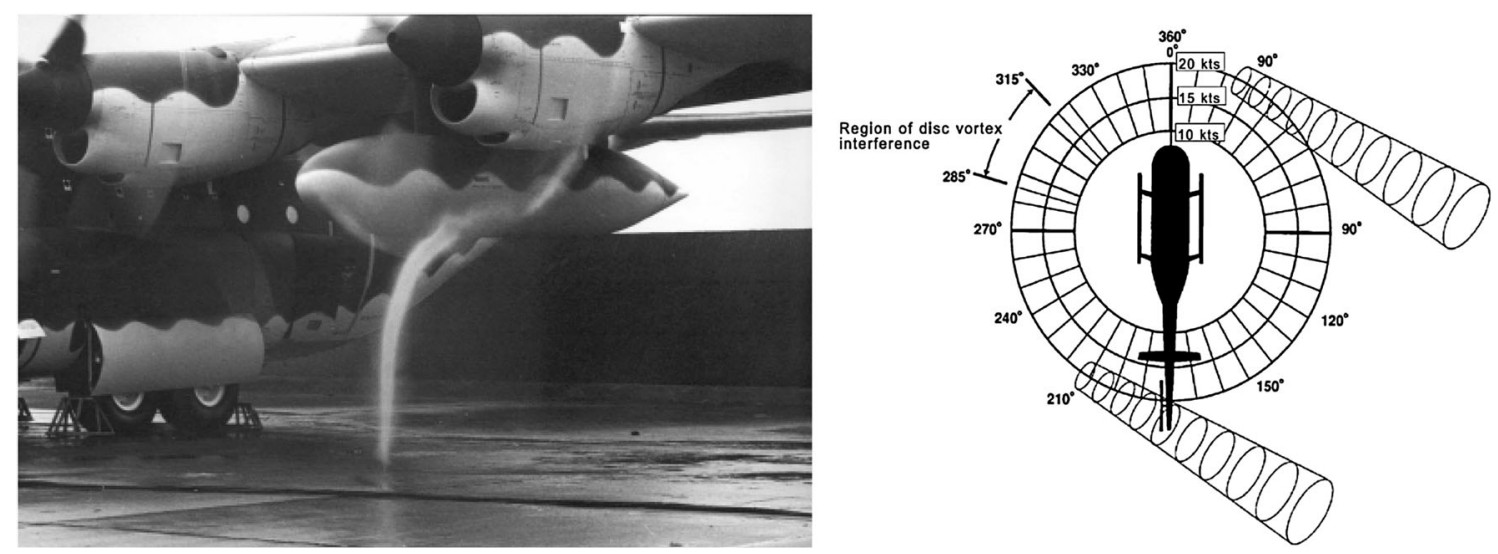

Fig. 1 Occurrence of vortices impingement on the blades. Left ground vortices generated from ground toward the blades of C130 [8]; right vortex shed from the main rotor of helicopter colliding with the tail rotor [9]

occur between the rotor and stator [5, 6]: the vortex shed from the rotor collides with the downstream stator [5]; the secondary vortex in the stator wake impinges on the rotor [6]. Blade-vortex interaction can also be observed when the vortex shed from the main rotor of helicopter collides with the tail rotor [7], as shown on the right-hand side of Fig. 1.

The presence of a concentrated vortex in the incoming flow leads to a non-uniform and distorted inflow into the engine [10], to an impulsive force on the blade [11] and to noise generation $[12,13]$. The ground vortex influence on a turbofan engine inflow has been studied in terms of flow distortion. It has been reported that the engine inflow distortion increases as the inflow vortex strength increases [14]. The thrust of the tail rotor of a helicopter increases when the rotation direction of the tip vortex from the main rotor has a direction opposite to the tail rotor, and vice versa for the co-rotating tail rotor [15]. Fluctuating pressure due to upstream vortices measured near the leading edge of a rotating blade showed impulsive features due to BVI, resulting in an increase in far field noise by $5-15 \mathrm{~dB}$ [12].

Although there are only few studies on vortex development after collision with rotating blades, studies on the vortex development after collision with a steady wing have been conducted more extensively [16, 17]. A streamwise vortex impinges below the pressure side of the zero loaded wing with symmetrical airfoil is studied in [17]. The vortex maintains the core structure downstream the trailing edge of the wing. The wake of the wing triggers the turbulent decay of the vortex core and induces the decrease of vortex strength and increase of vortex dimension further downstream [16]. The vortex development after collision with the blades is of interest for the concern of influence on the next stage of compressor of a turbofan engine, and the possibility of entering the intake of a turboprop engine.
The interaction between a single off-axis vortex and a propeller is investigated in this paper. The objectives of the study include two aspects: first, the propeller performance, as it is influenced by the vortex, in terms of thrust and torque (also termed the out of plane force and moment components); second, the development of the vortex before and after the propeller.

\section{Test rigs and parameters}

\subsection{Wind tunnel, propeller rigs, and vortex generator}

The tests were carried out in a low-speed and closed-loop open-jet wind tunnel in Delft University of Technology, the test section configuration of which is shown in Fig. 2. The wind tunnel has a maximum speed of $35 \mathrm{~m} / \mathrm{s}$ and an octagonal working section with dimension of $2.85 \mathrm{~m} \times 2.85 \mathrm{~m}$. The inflow velocity chosen in the experiments is $19.2 \mathrm{~m} / \mathrm{s}$

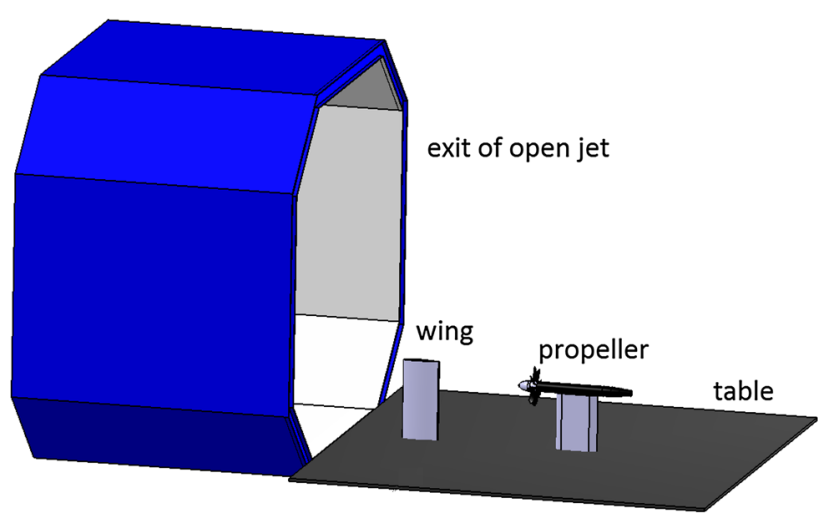

Fig. 2 Experimental setup of the propeller wing combination in the Open-Jet Facility (OJF) at Delft University of Technology 
which is below the maximum speed of the wind tunnel, because the density of particles for PIV measurement becomes low if the wind speed goes too high in this wind tunnel. A ground board is used which is aligned with the bottom of the tunnel exit.

The diameter of the eight-bladed propeller is $0.304 \mathrm{~m}$, and the blade angle at $3 / 4 R$ is set to $41^{\circ}$ which represents the take-off condition for a propeller aircraft. The total length of the model is $0.904 \mathrm{~m}$, including spinner, hub, and nacelle. The propeller is driven by the TDI 1999 pneumatic motor, and its maximum rotating speed is 22,000 rpm.

The vortex generator is a truncated two-dimensional DU96-W-180 airfoil at zero slip angle. The airfoil, designed for wind turbines, is used, because it was available; no specific considerations entered the choice. The span of the lifting surface is $1 \mathrm{~m}$, and chord length is $0.25 \mathrm{~m}$; and it is positioned 3.2 chord lengths upstream the propeller. The vortex is generated from the tip of the lifting surface at positive incidence angle. Although the vortex with opposite circulation can be generated at negative incidence angle, the profile of the vortex axial flow velocity changes as the airfoil is not symmetrical. To generate a vortex with opposite circulation and maintain the same axial velocity profile, the other end of the lifting surface is used as the other vortex generator. Therefore, there are two vortex generators of the two ends of the lifting surface in our study: one generates the vortex co-rotating with the propeller, and the other one generates the contra-rotating vortex. To adjust the vortex impinging position on the propeller, the wing can be moved in the transverse direction and spanwise direction.

\subsection{PIV setup}

To obtain insight into the development of the vortex, velocity measurements were performed in two planes perpendicular to the propeller axial direction. Three component, two-dimensional (3C-2D) PIV measurements were performed using two LaVision Imager Pro LX 16M cameras (CCD sensor of $4870 \times 3246$ pixels, 12 bit resolution, $7.4 \mathrm{~m}$ pixel pitch), and a Quantel Evergreen 200 laser (dual pulsed Nd:YAG laser, $200 \mathrm{~mJ}$ energy per pulse); the illumination is provided from the side of the propeller. The flow was seeded with micron-sized water-glycol particles produced by a SAFEX Twin Fog Double Power smoke generator inserted into the settling chamber.

Two planes normal to the propeller axis are investigated: one upstream of the propeller was positioned at $X /$ $R=-0.20$, where the propeller leading edge is at $X=0$ (the coordinate system is defined in Fig. 4), which is on the left of Fig. 3, and one downstream of the propeller $(X /$ $R=0.13$ ), which is on the right of Fig. 3. For PIV arrangement 1 , one camera with forward scattering of the laser sheet is set at $42^{\circ}$ with respect to the propeller axis, and the other camera with backward scattering of the laser sheet is set at $44^{\circ}$. For PIV arrangement 2, one camera with forward scattering of the laser sheet is set at $41^{\circ}$, and the other camera with backward scattering of the laser sheet is set at $43^{\circ}$.

\subsection{Definitions and parameters}

The coordinate system for the flow around the propeller is defined on the left-hand side of Fig. 4, and the circumferential position of the blade is defined on the right-hand side of Fig. 4. The vortex impinging position is located at $Y=$ $0, Z=0.75 R$ as viewed on plane $X / R=-0.20$. In this paper, all the PIV data reduction is performed at phase angle $\Psi=22.5^{\circ}$, in which condition the two blades (e.g., blade 4 and blade 5 shown in Fig. 4) are located symmetrically on the two sides of the impinging vortex. The vortex core does not impinge on the blade at this phase angle, which is a typical case for studying vortex-blade interaction. The study of vortex properties during impinging on the blade will be performed later. The result from balance measurement is averaged over all phase angles, because only the time-averaged forces and moments are acquired.

To study the vortex impact on the propeller, out of plane force and moment measurements are conducted to establish a correspondence between the propeller performance and the vortex strength. The parameter that determines the propeller loading is the advance ratio, defined as:
Fig. 3 PIV measurement setup for the field of view (FOV) in front of the propeller (left) and behind the propeller (right)
PIV arrangment 1

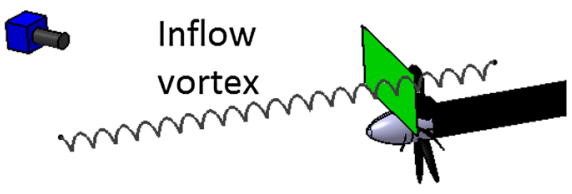

PIV arrangement 2

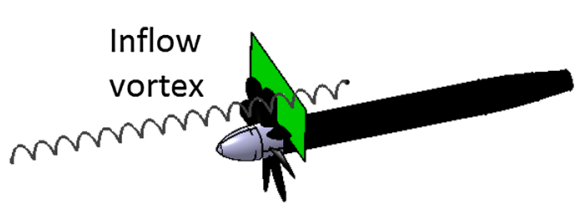

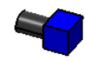


Fig. 4 Left definition of coordinate system; right definition of blade phase angle (reference line goes through $42 \%$ of chord length at 0.75 $R$ position), viewed from the front
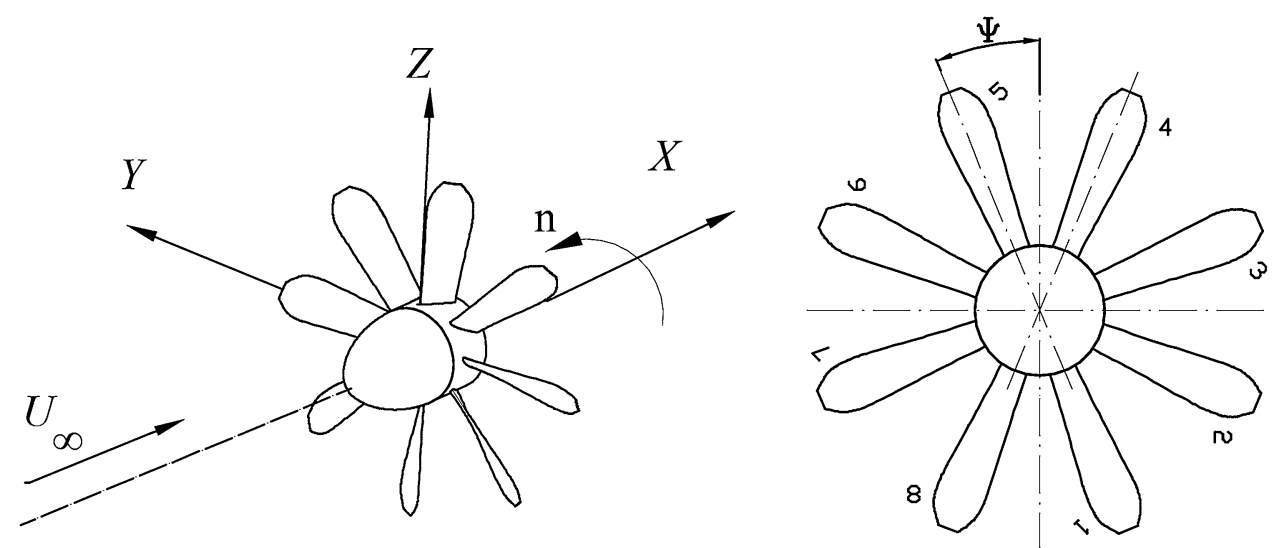

$J=U_{\infty} / n D$.

For a propeller with axisymmetric inflow, the time-averaged inflow velocity in axial direction can be predicted by a modified actuator disk model [18], which assumes that the pressure jump across the disk is generated by the upstream suction of the propeller; the slipstream is immediately expanded to the free stream pressure. The equivalent inflow velocity in the propeller plane is assumed uniform both in the azimuthal and radial directions,

$U_{\text {eq }}=U_{\infty} \sqrt{1+8 \cdot T_{C} / \pi}$,

where $T_{c}$ is the thrust coefficient, defined as

$T_{c}=T / \rho U_{\infty}^{2} D^{2}$,

where $T$ is the propeller thrust, and $\rho$ is the air density. The torque coefficient is defined as

$Q_{c}=\frac{Q}{\rho U_{\infty}^{2} D^{3}}$,

where $Q$ is the torque.
The circulation in the impinging vortex core region, $\Gamma$, is normalized by the diameter and inflow velocity of propeller

$\Gamma_{\text {imp,cor }}=\Gamma /\left(U_{\text {eq }} * D\right)$.

\section{Results}

\subsection{Profile of wing tip vortex}

The vortex generated from wing tip is analyzed from the PIV measurement and shown on the left-hand side of Fig. 5. The swirling flow covers the whole field of view of PIV measurement $\left(2 R^{*} 1 R\right)$. The streamline plot is colorcoded by vorticity, which is normalized by the propeller inflow velocity and diameter. Even though the propeller is not present in the flow during the measurement of wing tip vortex, the propeller inflow velocity is still selected for normalization on the vorticity for consistence with the following sections in which there is propeller in the flow.
Fig. 5 Left flow field of wing tip vortex; right transverse velocity distribution $\left(U_{Y}\right)$ at cross section through the vortex center

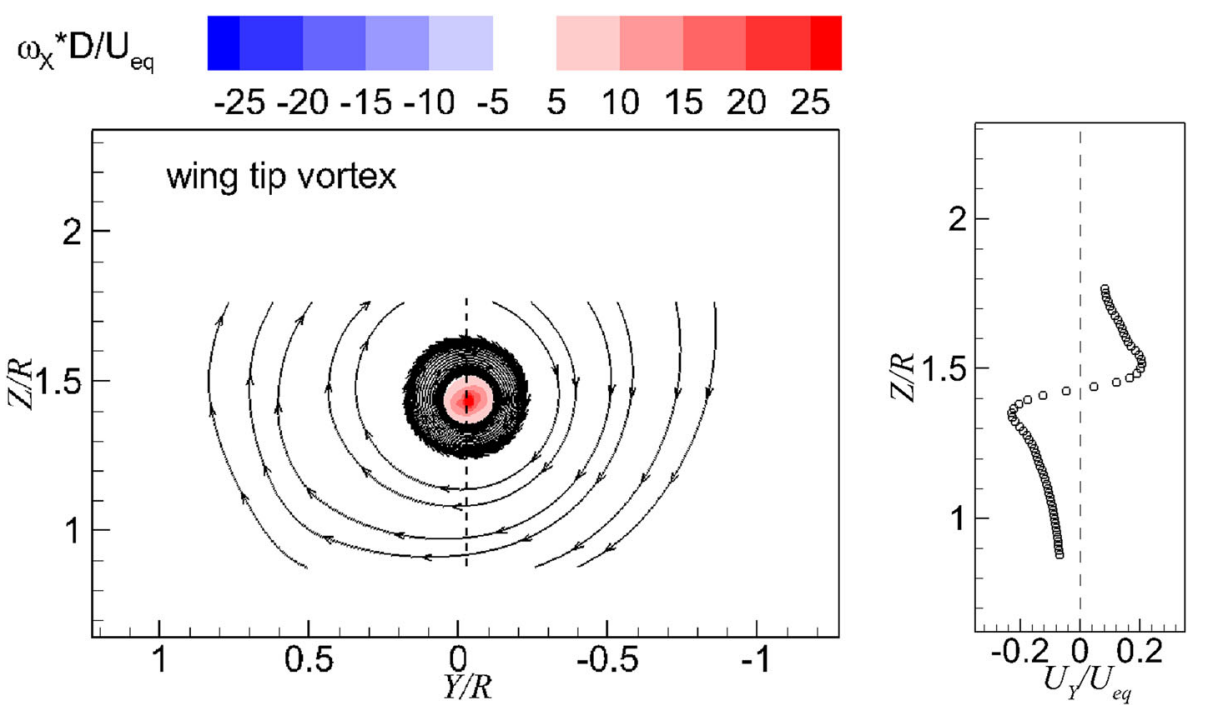


The distribution of velocity $\left(U_{Y}\right)$ along the line through the vortex center (shown by the dashed line on the left-hand side of Fig. 5) is shown on the right-hand side of Fig. 5. $Y$-component velocity is zero at the vortex center, and it has a symmetrical profile with respect to its center position. From the center to the outboard of vortex, $Y$-component velocity increases until reaching the maximum value, and then it decreases.

The circulation of the core of wing tip vortex at plane $X / R=-0.20$ is defined as the parameter to characterize the impinging vortex strength, $\Gamma_{\text {imp,cor }}$. The center of the vortex at plane $X / R=-0.20$ is also defined as the impinging position of the vortex.

\subsection{Flow field upstream the propeller}

Two views of the incoming flow of propeller involving vortex at plane $X / R=-0.20$ are shown in Fig. 6 . The figure on the left-hand side illustrates the flow field with a vortex with the same rotating direction as the propeller, and the figure on the right-hand side shows the flow field with a contra-rotating vortex.

The test conditions in terms of thrust coefficients are $T_{c}=1.54$ and $T_{c}=1.70$ for co-rotating vortex and contrarotating vortex, respectively. The maximum value of axial flow velocity in the measurement domain, $\left(U_{X} / U_{\mathrm{eq}}\right)_{\max }$, is around 0.75 ; the maximum value of tangential flow velocity in the measurement domain, $\left(U_{\tan } / U_{\text {eq }}\right)_{\max }$, is around 0.3 . The swirl velocity in the propeller inflow is in the same order of the axial flow velocity.

The vortical flow field features a node (N1) and a saddle point (S1). The saddle point is induced by the opposite direction of propeller-induced radial flow and tangential flow of the vortex. The saddle point is located on the righthand side for the co-rotating vortex and the left-hand side for the contra-rotating vortex.

\subsection{Flow field downstream the propeller}

The flow field downstream the propeller is shown in Fig. 7, color-coded by axial vorticity. The maximum axial flow velocity in the slipstream is $\left(U_{X} / U_{\mathrm{eq}}\right)_{\max }=1.13$, and the maximum tangential flow velocity in the slipstream is $\left(U_{\tan } / U_{\mathrm{eq}}\right)_{\max }=0.79$. The tangential velocity in the slipstream features the same direction as the propeller rotation.

Vorticity is positive in the blade tip region and negative in the blade wake region. The vortical flow in the blade tip region cannot be observed because of the high helicity of the tip vortex at low advance ratio. The vorticity peak found in the propeller inflow can still be recognized in the slipstream (located inside the black dashed circle shown in Fig. 7), but the flow pattern of focal lines around the vortex centre does not exist anymore, which is due to the main stream of the tangential velocity of the slipstream. It is also noted that the magnitude of streamwise vorticity in the wake of the contra-rotating vortex is larger than that of the co-rotating vortex. This is due to the increase of spanwise velocity gradient on the suction side and pressure side of the blade [17]. For a co-rotating vortex, the vortex induces outward spanwise velocity on the pressure side of blade 4 (shown in Fig. 4) and inward spanwise velocity on the suction side of blade 5 . As a result, the streamwise vorticity magnitude in the wake decreases, and vice versa for the contra-rotating vortex.
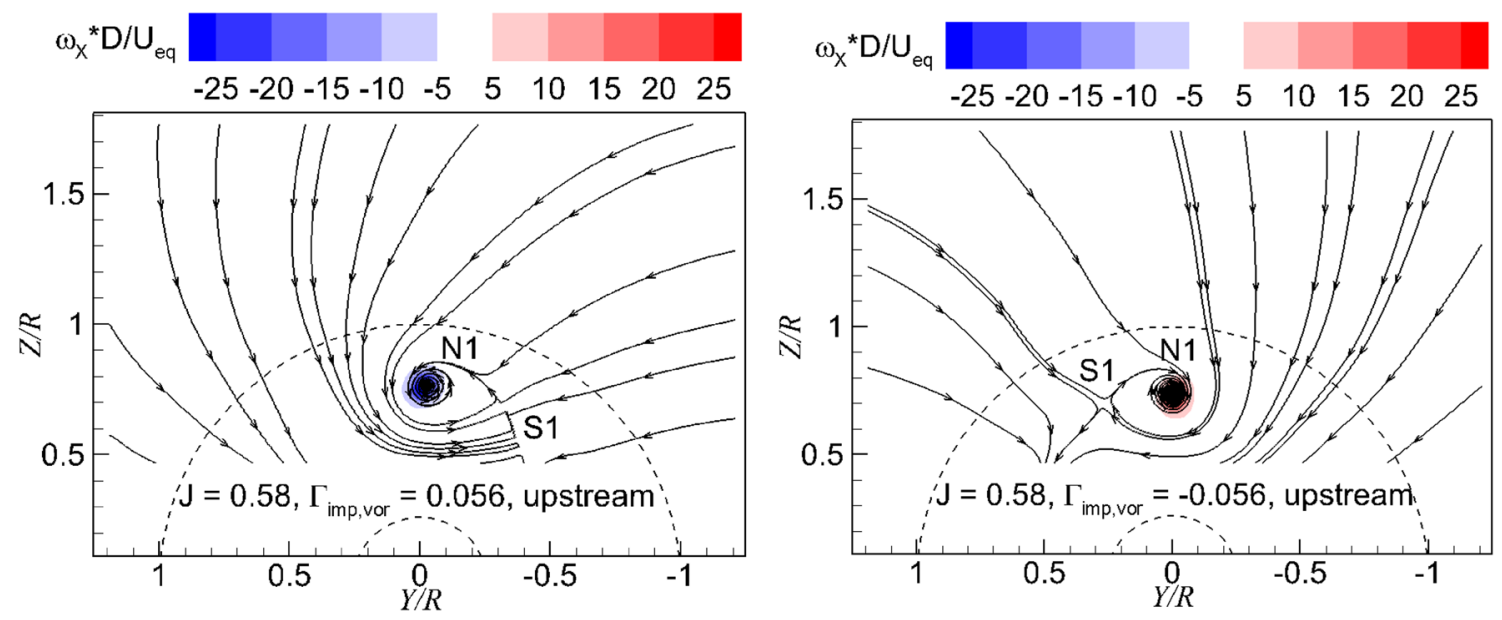

Fig. 6 Flow field upstream the propeller. Left flow field involving co-rotating vortex, $T_{c}=1.54$; right flow field involving contra-rotating vortex, $T_{c}=1.70, \Psi=22.5^{\circ}$ 

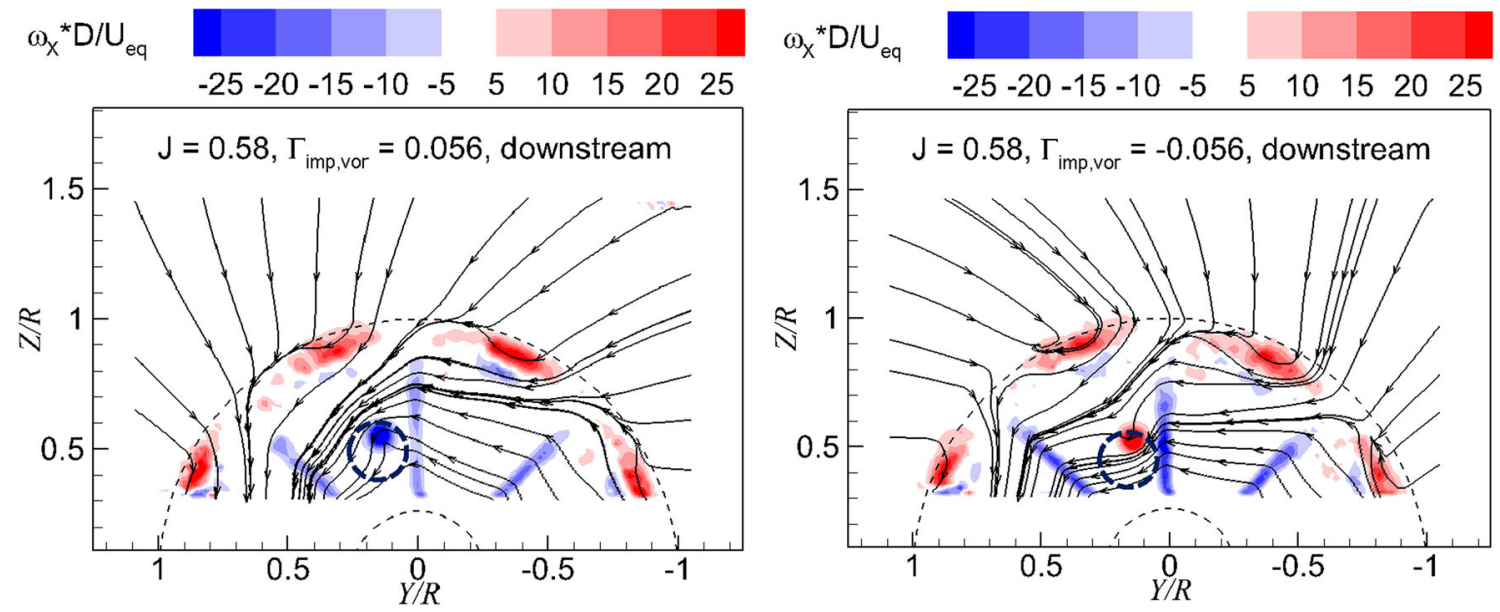

Fig. 7 Flow field downstream of propeller. Left the flow field involving co-rotating vortex, $T_{c}=1.54$; right flow field involving contra-rotating vortex, $T_{c}=1.70 . \Psi=22.5^{\circ}$

\section{Data analysis}

\subsection{Propeller impact on the vortex}

\subsubsection{Vortex strength and dimension}

The vortex development from upstream to downstream of the propeller is investigated in this section. The tangential velocity distribution of the vortex in the radial direction is shown in Fig. 8. The vortex core radius is defined by the distance from the vortex center to the position, where the maximum tangential velocity is achieved. The axial flow velocity increases from upstream to downstream the propeller due to the propeller suction and the vortex is stretched; therefore, the vortex core radius decreases, as it goes through the propeller. For the co-rotating vortex, the tangential velocity downstream is higher compared with upstream which is shown on the left-hand side of Fig. 8.
For the contra-rotating vortex, the tangential velocity follows the same trend as the co-rotating vortex, nevertheless the tangential velocity becomes approximately the same as the upstream from radial position $r / R=0.09$, which is shown on the right-hand side of Fig. 8 . The reason is the negative vorticity in the blade wake, which is opposite with the inflow vorticity, as shown in Fig. 7.

\subsubsection{Vortex position}

The positions of the vortices in the measurement planes upstream and downstream the propeller are shown in Fig. 9. The averaged position of co-rotating vortices is located at $[0,0.73 R]$ upstream the propeller and $[0.14 R$, $0.53 R]$ downstream the propeller. The averaged position of contra-rotating vortices is located at $[0,0.76 R]$ upstream the propeller and $[0.14 R, 0.57 R]$ downstream the propeller. In the radial direction, the vortex moves inward as
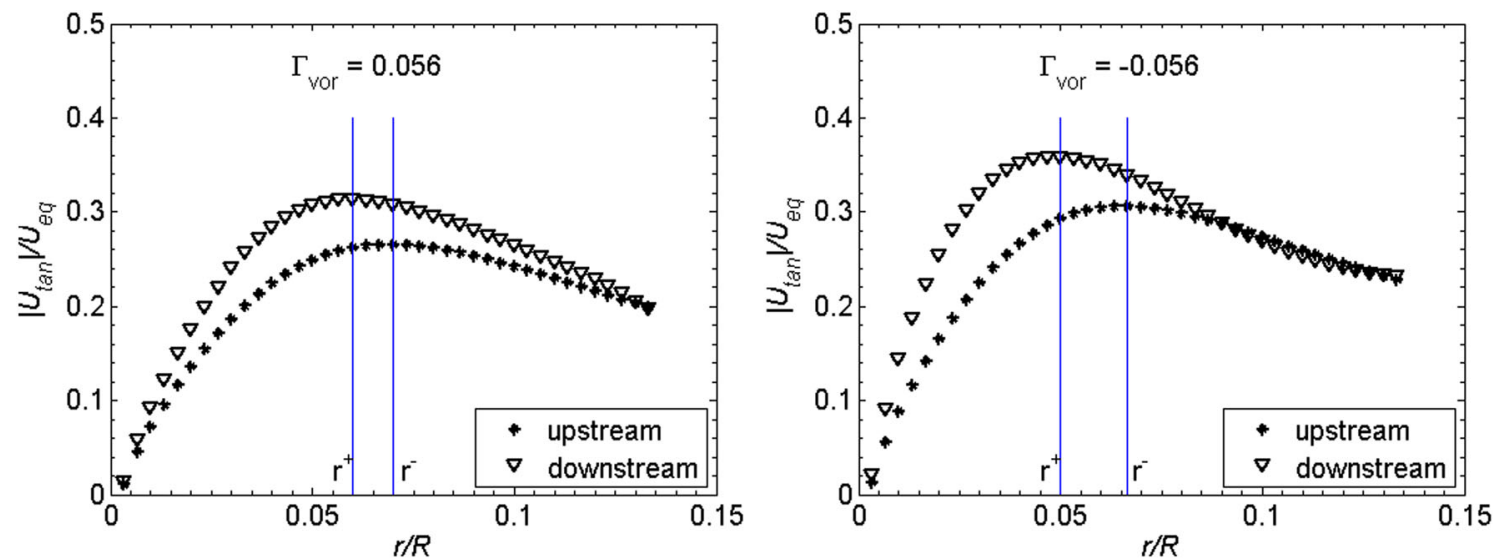

Fig. 8 Tangential velocity of the incoming vortex upstream and downstream the propeller $\left(r^{-}\right.$is the vortex core radius upstream the propeller, and $r^{+}$is the vortex core radius downstream the propeller). Left co-rotating vortex; right: contra-rotating vortex. $\Psi=22.5^{\circ}$ 

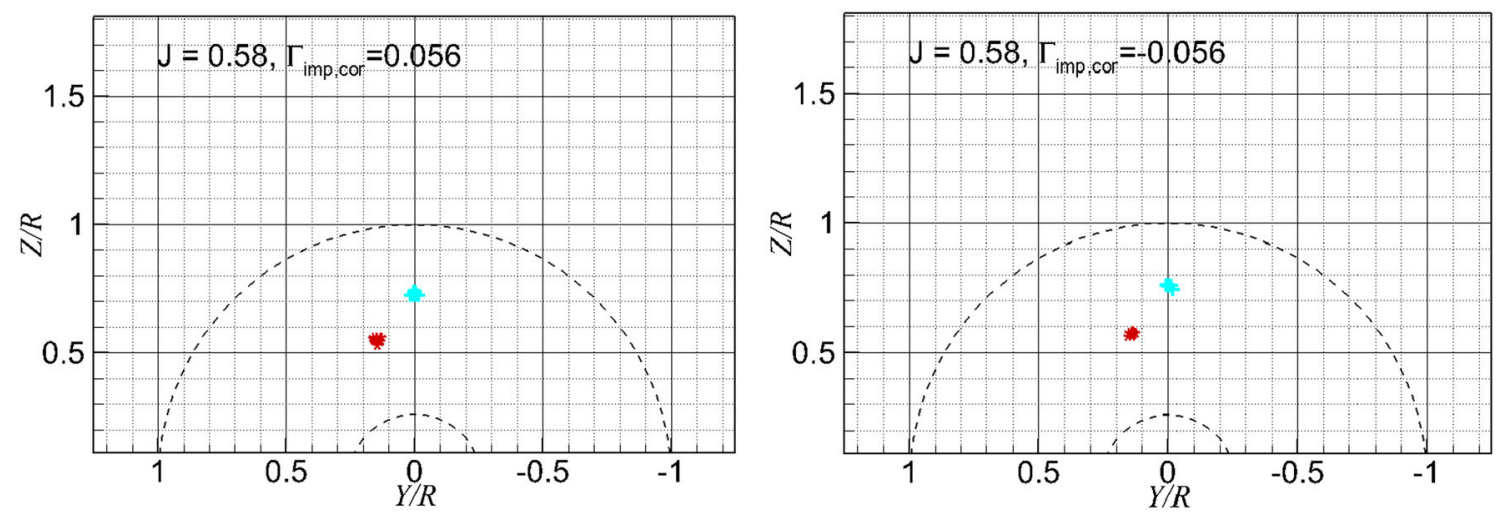

Fig. 9 Positions of vortices upstream of the propeller (blue '+') and downstream of the propeller (red '*'). $\Psi=22.5^{\circ}$

the vortex goes through the propeller; in the circumferential direction, the vortex movement follows the same direction as the rotation of the slipstream. The displacement distance it covers is around $0.2 R$ at the measurement plane. The meandering of vortices is not strong at one fixed phase angle of propeller.

The inward motion in radial direction of the vortex may mainly be ascribed to the contraction of the streamtube. The circumferential movement of the vortex is mainly due to the tangential velocity in the slipstream, i.e., the swirl.

\subsection{Impact of the vortex on the propeller}

\subsubsection{Influence of the vortex on the tangential velocity of propeller inflow}

The investigation of vortex impact on the performance of propeller is conducted by analyzing the propeller local inflow, i.e., tangential velocity and axial velocity, and the out of plane force and moment of the propeller.
Figure 10 shows the tangential velocity upstream the propeller, in which the red color represents the same direction as the propeller rotation, and vice versa for the blue color. A co-rotating vortex in the inflow induces a positive tangential velocity in the outboard region of the propeller projection area and a negative tangential velocity in the inboard region, the opposite occurs for the contrarotating vortex.

The tangential velocity of the propeller inflow due to vortex impact can be analyzed from the model shown in Fig. 11. The black solid curve represents the propeller (which is centered at ' $\mathrm{P}$ '); the arrow shows the rotation direction of propeller. The black dashed curve represents a streamline of the vortex (which is centered at ' $\mathrm{V}$ '); the arrow shows the tangential velocity direction of vortex. There is a blue dashed circle with the diameter of the distance between propeller center ' $\mathrm{P}$ ' and vortex center ' $\mathrm{V}$ '. Two lines, e.g., VA and AP from point ' $A$ ' on blue dashed circle to the centers of ' $\mathrm{P}$ ' and ' $\mathrm{V}$ ', respectively, are perpendicular. The tangential velocity of the vortex, which
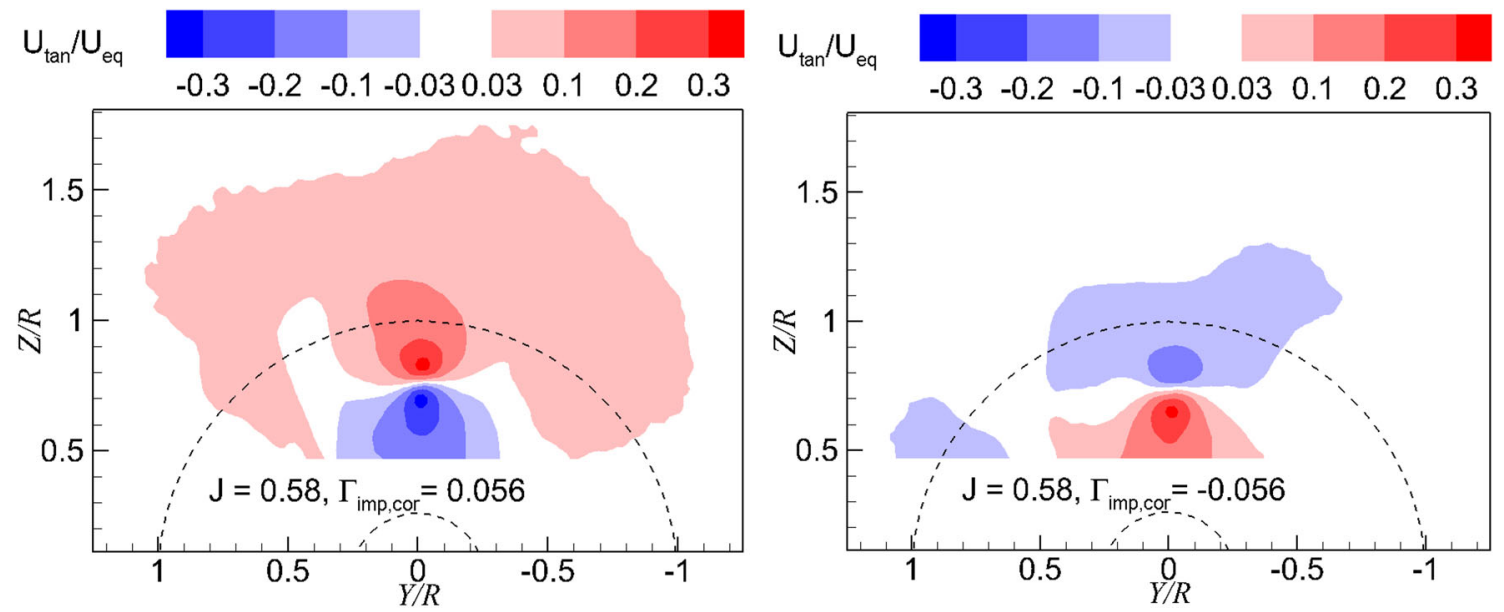

Fig. 10 Tangential velocity in the upstream of propeller. Left co-rotating vortex, $T_{c}=1.54$; Right contra-rotating vortex, $T_{c}=1.70 . \Psi=22.5^{\circ}$ 


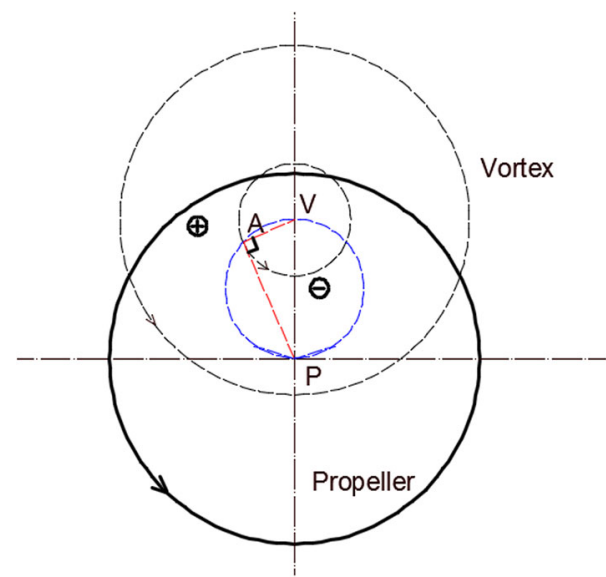

Fig. 11 Vortex tangential velocity effect on different regions of propeller (co-rotating vortex)

is in the same direction of $\overrightarrow{\mathrm{AP}}$, has zero component on the tangential velocity of propeller which in the direction of $\overrightarrow{\mathrm{VA}}$. The blue dashed circle is the domain boundary of the propeller tangential velocity: for a co-rotating vortex, the tangential velocity of propeller inflow is positive outside the blue dashed circle, and negative inside the blue dashed circle.

It is obvious that the area inside the blue circle is less than the remaining area of the propeller disk, so the resultant tangential velocity of the propeller inflow is consistent with the region outside the blue dashed circle. As a result, the tangential velocity of the propeller inflow is positive for the co-rotating vortex, and vice versa for the contra-rotating vortex.

The effect of the induced tangential velocity on the propeller is similar to the effect of the swirling flow of the propeller slipstream on the wing; it is reported that the upwash region on the wing due to the propeller slipstream induces an increase of the lift, and vice versa for the downwash region [19]. Due to the induced velocity of the vortex on the propeller inflow, there is a loading increase or decreases depend on the upwash or downwash effects, respectively. The integral effect of these effects will be evaluated by the balance measurement, as shown in Sect. 4.2.3.

\subsubsection{Influence of the vortex on the axial velocity of propeller inflow}

The axial velocity distribution in the propeller inflow is shown in Fig. 12. The axial velocity inside the propeller disk is higher than in the outer region because of the propeller suction. The axial velocity in the vortex core region has a wake shape when the vortex co-rotates with the propeller, and the contra-rotating vortex has a jet like shape axial velocity. This difference is probably due to the different shape of wing tip end. Compared with influenced area in the propeller inflow of tangential velocity, the axial flow of vortex has less influenced area.

\subsubsection{Performance of propeller}

The thrust and torque coefficients are shown in Fig. 13 at different strengths of vortex. The measurements are conducted at the same impinging position of the vortex, $\mathrm{r} / R=$ 0.75 and the same advance ratio, $J=0.58$. We consider a reference condition that where no vortex is impinging on the propeller: $\Gamma_{\text {imp,cor }}=0, T_{c}=1.62, Q_{c}=0.43$.

When the circulation is negative, both $T_{c}$ and $Q_{c}$ are higher than the reference point; when circulation is positive, they are both lower than the reference point. As the circulation increases from negative to positive, both $T_{c}$ and $Q_{c}$ decrease monotonically.
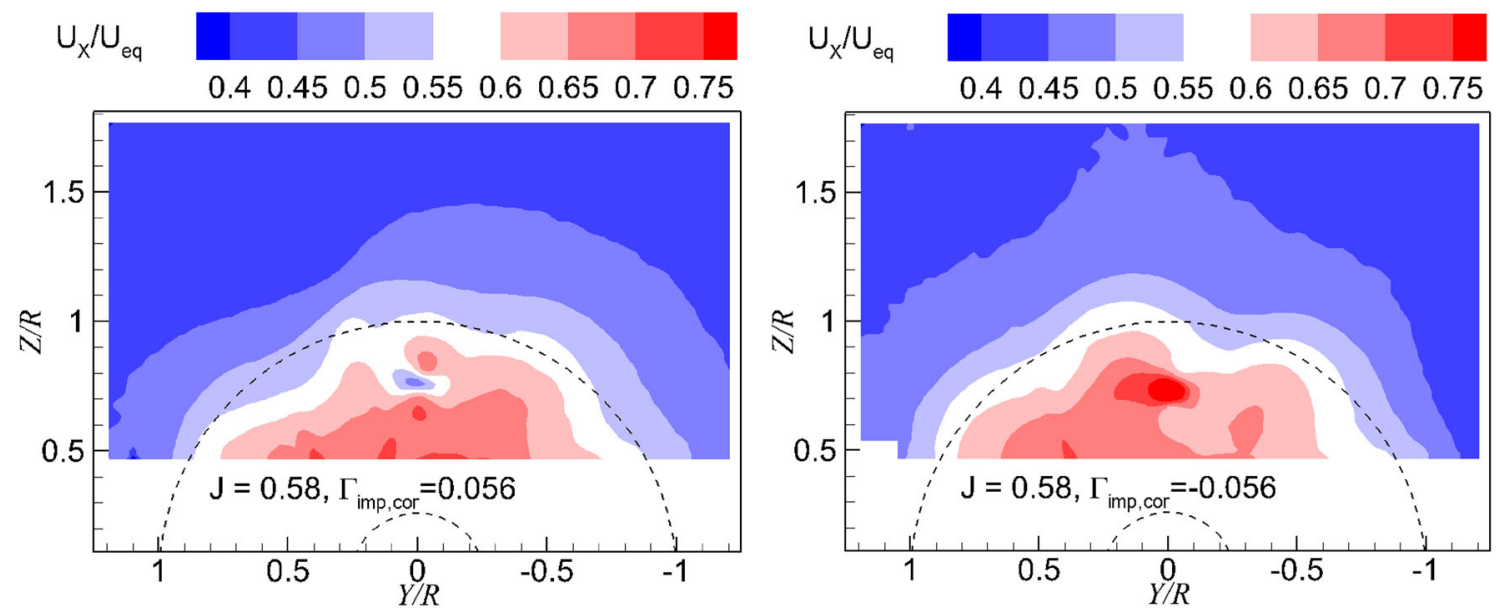

Fig. 12 Axial velocity upstream of the propeller involving co-rotating vortex $($ left $)$ and contra-rotating vortex $(r i g h t) . \Psi=22.5^{\circ}$ 

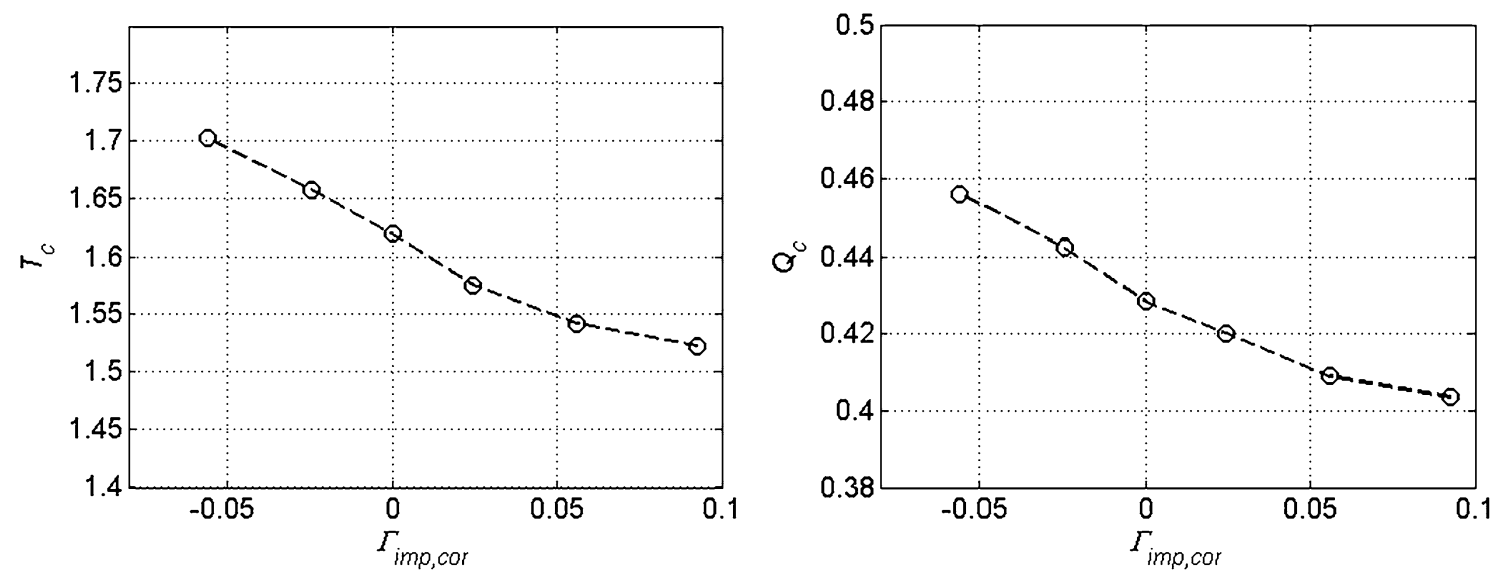

Fig. 13 Propeller performance dependence on the vortex strength, $J=0.58$

It is shown in Fig. 11 that the induced tangential velocity in the propeller inflow has the same direction as the propeller rotation when the vortex is co-rotating. Consequently, the propeller thrust and torque coefficients decrease, because the effective rotating speed decreases. It is also shown in Fig. 12 that the axial flow velocity in the vortex core decreases when the vortex is co-rotating, resulting in an increase of thrust and torque coefficients. These two velocity components have opposite effect on the propeller performance. However, the trend of the propeller performance is consistent with the impact of tangential velocity of propeller inflow. Thus, it can be concluded that the tangential velocity of the vortex dominates the impact on the propeller performance.

\section{Conclusions}

Propeller and vortex interaction is studied by a streamwise wing tip vortex impinging on the propeller. A series of vortices impinging on the propeller are investigated at one propeller advance ratio, $J=0.58$, and one vortex impinging position, $r / R=0.75$. Data reduction of PIV measurement is performed at vortex impinging phase angle, $\Psi=22.5^{\circ}$. Data reduction of balance measurements is performed by averaging all the phase angles.

The tangential velocity of the vortex dominates the effect on the propeller performance, and the axial velocity of the vortex plays a less important role. The propeller thrust and torque coefficients increase when the incoming vortex has the opposite direction of the propeller rotation, and vice versa for the co-rotating vortex. As the circulation of the impinging vortex increases, the out of plane force and moment decrease.

Along with the stream-tube contraction and introduction of swirl from upstream of the propeller to downstream of the propeller plane, the impinging vortex is displaced and stretched. The vortex moves radially inward and circumferentially in the same direction of the slipstream for both co-rotating and contra-rotating vortex. In the process of transiting through the propeller plane, the vortex radius decreases and its tangential velocity in the vortex core increases. The vortex, despite the stretching and displacement, will remain a concentrated feature available for interactions with further stages of the propulsor.

Although the thrust and torque coefficient of the propeller are measured and both show increases due to a contra-rotating vortex at a given advance ratio, the dynamic loading on the propeller needs to be evaluated for the concern of tonal noise and structural fatigue issues. Because the current balance measurement can only obtain the time-averaged loadings on the propeller, the ongoing unsteady CFD research by the authors is the dynamic loading on the individual blade, which will generate detailed understanding on the time-dependent loading of the blade, pressure fluctuation on the blade, etc.

Open Access This article is distributed under the terms of the Creative Commons Attribution 4.0 International License (http://crea tivecommons.org/licenses/by/4.0/), which permits unrestricted use, distribution, and reproduction in any medium, provided you give appropriate credit to the original author(s) and the source, provide a link to the Creative Commons license, and indicate if changes were made.

\section{References}

1. Yang, Y., Sciacchitano, A., Veldhuis, L., and Eitelberg, G.: Experimental investigation of propeller induced ground vortex under headwind condition. In: AIAA Aviation, Atlanta. AIAA, pp. 2014-2308 (2014)

2. Stuermer, A., Yin, J.: Low-speed aerodynamics and aeroacoustics of CROR propulsion systems. In: 15th AIAA/CEAS Aeroacoustics Conference (30th AIAA Aeroacoustics Conference), Miami, 2009 
3. Marquez, C.O., Steiner, S.: Aerodynamic installation effects of CROR engines at take-off configurations. In: 52nd Aerospace Sciences Meeting. AIAA SciTech, Maryland (2014)

4. De Siervi, F., Viguier, H.C., Greitzer, E.M., Tan, C.S.: Mechanisms of inlet-vortex formation. J. Fluid Mech. 124, 173-207 (1982)

5. Donlisk, A.T.: A theory of vortex-surface collisions. In: 2nd AIAA Theoretical Fluid Mechanics Meeting, Albuquerque, NM, USA, 1998

6. Binder, A., Forster, W., Mach, K., Rogge, H.: Unsteady flow interaction caused by stator secondary vortices in a turbine rotor. J. Turbomach. 109, 251-256 (1987)

7. Leverton, J.W., Pollard, J.S., Wills, C.R.: Main rotor wake/tail rotor interaction. Vertica 1, 213-221 (1977)

8. Campbell, J., Chambers, J.: Natural visualization of aircraft flow fields. NASA SP-514, 1994

9. Aviation occurrence report: loss of directional control. Report number: A98W0056, Highland helicopters Ltd, 1998

10. Murphy, J.P., MacManus, D.G.: Inlet ground vortex aerodynamics under headwind conditions. Aerosp. Sci. Technol. 15, 207-215 (2011)

11. Nagahara, T., Sato, T., Okamura, T.: Effect of the submerged vortex cavitation occurred in pump suction intake on hydraulic forces of mixed flow pump impeller. In: Brennen, C., Ceccio, S., Arndt R., (eds.) CAV 2001: 4th Intl Symp. on Cavitation, Pasadena, California, 2001
12. Ahmadi, A.R., Beranek, B., Newman Inc.: An experimental investigation of blade vortex interaction at normal incidence. AIAA-85-0003, Reno, Nevada, 1985

13. Povinelli, F.P., Dittmar, J.H., Woodward R.P.: Effects of installation caused flow distortion on noise from a fan designed for turbofan engines. NASA TN D-7076, 1972

14. Murphy, J.P., MacManus, D.G., Sheaf, C.T.: Experimental investigation of intake ground vortices during take-off. AIAA J. 48(3), 688-701 (2010)

15. Fletcher, T.M., Brown, R.E.: Helicopter tail rotor thrust and main rotor wake coupling in crosswind flight. J. Aircr. 47(6), 2136-2148 (2010)

16. Daccache, E., Lee, T.: Effect of a winglet on normal blade-vortex interaction. J. Aircr. 44(1), 264-273 (2007)

17. Wittmer, K.S., Devenport, W.J.: Effects of perpendicular bladevortex interaction, part 1: turbulence structure and development. AIAA J. 37(7), 805-813 (1999)

18. Yang, Y., Ragni, D., Veldhuis, L., Eitelberg, G.: Propeller Induced Ground Vortex. ICAS, Brisbane (2012)

19. Ferraro, G., Kipouros, T., Savill, A.M.: Propeller-wing interaction prediction for early design. In: 52nd Aerospace Science Meeting, Mayland, 2014 